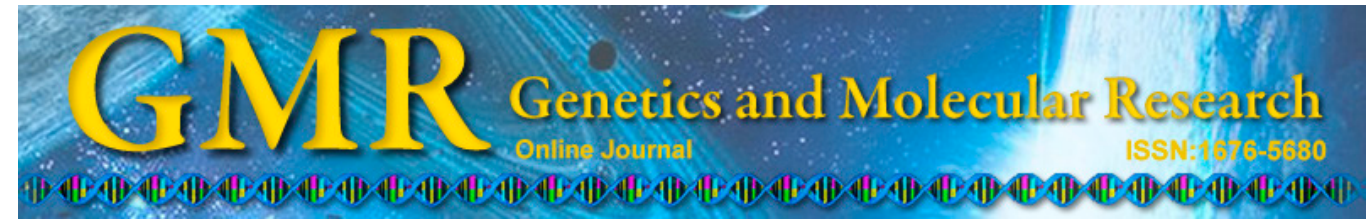

\title{
Association between $p 53$ codon 72 polymorphisms and clinical outcome of nasopharyngeal carcinoma
}

\author{
M.L. Li ${ }^{1,2}$, Y. Dong ${ }^{3}$, Y.Z. Hao ${ }^{2}$, N. Xu' ${ }^{2}$, F.L. Ning ${ }^{2}$, S.S. Chen ${ }^{2}$ and J.M. Yu ${ }^{4}$ \\ ${ }^{1}$ Division of Medicine, Shandong University, Jinan, Shandong, China \\ ${ }^{2}$ Oncology Department of Binzhou Medical University, Binzhou, China \\ ${ }^{3}$ Laboratory Department of Binzhou Medical University, Binzhou, China \\ ${ }^{4}$ Department of Radiation Oncology, Shandong Cancer Hospital and Institute, \\ Jinan, China \\ Corresponding author: J.M. Yu \\ E-mail: dy9712@yeah.net
}

Genet. Mol. Res. 13 (4): 10883-10890 (2014)

Received January 16, 2014

Accepted June 16, 2014

Published December 19, 2014

DOI http://dx.doi.org/10.4238/2014.December.19.9

\begin{abstract}
We conducted a cohort study to investigate whether polymorphisms in $p 53$ at codon 72 are associated with tumor response and survival time of advanced nasopharyngeal carcinoma (NPC) patients treated with radiotherapy. The study population included 127 subjects with NPC who were enrolled at Binzhou Medical University between September 2008 and December 2009. Cox proportional hazard regression was used to assess the association between polymorphisms in the $p 53$ gene and progression-free survival (PFS) and overall survival (OS) of NPC patients. During the follow-up period, 42 patients died and 72 patients showed progression at the end of the study. Of the 127 patients, median PFS was $22.5 \pm 1.2$ months (1-36 months), and the median OS time was $28.2 \pm 1.1$ months (2-36 months). The $p 53$ codon 72 Pro/Pro genotype was associated with a
\end{abstract}


longer median PFS time of 30.3 months compared with 18.2 months for patients with Arg/Arg variants. Moreover, the $p 53$ codon 72 Pro/ Pro genotype was associated with a longer median OS time of 31.6 months compared with 25.8 months for those with Arg/Arg variants; the P value was marginally significant. We showed that variants in p53 codon 72 may be an independent predictor for PFS and OS of NPC patients.

Key words: Clinical outcome; Nasopharyngeal carcinoma; p53 codon 72

\section{INTRODUCTION}

Nasopharyngeal carcinoma (NPC) is a common malignancy in Southeast Asian populations, with an annual incidence of 3.9 cases per $10^{5}$ individuals (IARC, 2012). EpsteinBarr virus infection, tobacco smoking, alcohol consumption, and occupational exposure to wood dust, as well as high salt consumption, play an important role in NPC development (Hildesheim et al., 2001; Jia et al., 2010; Polesel et al., 2011; Fachiroh et al., 2012; Song and Yang, 2013). Because $80 \%$ of NPC cases are diagnosed late because of the deep location of the suspected tumor and non-specificity of initial symptoms, the survival of nasopharyngeal carcinoma is poor (Xi et al., 2013). For patients with stage II or III nasopharyngeal carcinoma, the 5 -year overall survival (OS) rate is only approximately $45 \%$, despite aggressive concurrent chemoradiation therapy (Sun et al., 2013); however, lesions often develop distant metastases despite local control. Radiotherapy is the main treatment for locoregional NPC, but patients in the same tumor stages show different responses to radiotherapy and varying survival time (Xi et al., 2013; Sun et al., 2013). Therefore, genetic factors may influence the toxicity of chemotherapy and outcome of NPC.

TP53 is one of the most widely studied tumor suppressor genes and plays an important role in cell cycle regulation. Mutations in TP53 can disrupt cell cycle regulation, damaging its DNA-binding properties and transcription factor function and inducing aberrant cell proliferation. Inhibition of the p53 pathway can accelerate cancer progression and cause resistance to chemotherapy and radiotherapy (Malkin et al., 1992). p53 function can be altered by a common sequence polymorphism at codon 72 in the $p 53$ gene. Variations at codon 72 alter the activity of the p53 protein, as this polymorphism is located in the prolinerich domain of $\mathrm{p} 53$, which is necessary for the $\mathrm{p} 53$ protein to fully induce apoptosis (Thomas et al., 1999; Baptiste et al., 2002). Furthermore, patients with inoperable cancer carrying the p53 72Arg allele showed a higher response rate to chemotherapy and radiotherapy as well as longer survival time when compared with the $p 53$ 72Pro variant (Sullivan et al., 2004). This indicates that variations in $p 53$ are important for determining cellular sensitivity to chemotherapy and radiotherapy.

We conducted a cohort study to investigate whether polymorphisms in $p 53$ at codon 72 are associated with tumor response and survival time of advanced NPC patients treated with radiotherapy. 


\section{MATERIAL AND METHODS}

The study population included 127 subjects with NPC who were enrolled at Binzhou Medical University between September 2008 and December 2009. All patients were diagnosed with NPC by biopsy. Tumor stages were classified according to American Joint Committee on Cancer (AJCC)/International Union Against Cancer (UICC) criteria. Patients who had metastasis, other secondary tumors, or a history of other malignant neoplasm were excluded from the study. All patients were asked to provide $5 \mathrm{~mL}$ peripheral blood and informed consent. The Ethics Committee of the First Affiliated Hospital of the Binzhou Medical University reviewed and approved the study.

All patients received a complete physical examination, fiberoptic nasopharyngoscopy, magnetic resonance imaging, and chest X-ray, as well as abdominal imaging with ultrasound before receiving treatment. Megavoltage photons (6 MV) were taken to treat primary tumor and neck lymph nodes of all patients. Radiotherapy was administered 5 times per week with a dose of 2 Gy/day. The accumulated dosage of radiation was 68-72 Gy for the primary tumor, 60-62 for the neck lymph nodes, and 50 Gy for uninvolved areas. Concurrent chemotherapy was used for 41 patients $(31.8 \%)$, and $100 \mathrm{mg} / \mathrm{m}^{2}$ cisplatin was administered on days 1,22 , and 43 during radiotherapy.

All patients were followed-up until December 2012, with a median follow-up period of 22.4 months (3-36 months). All patients were followed-up by telephone or outpatient service every 2 months until death. The recurrence and distant metastases were diagnosed based on fiberoptic endoscopy, magnetic resonance imaging, and physical examination.

Progression-free survival (PFS) was defined as the time from the date of the radiotherapy to recurrence and distant metastases. OS was defined as the time from the date of radiotherapy start to the date of death or last clinical follow-up.

\section{Genotyping analysis}

Genomic DNA was extracted using the TIANamp Blood DNA Kit (Tiangen, Beijing, China) according to the manufacturer protocol. Genotyping of polymorphisms at codon 72 in the $p 53$ gene was conducted using a polymerase chain reaction (PCR)-restriction fragment length polymorphism (RFLP) method. The PCR primer pairs of the $p 53$ gene at codon 72 for PCR amplification were designed using the Sequenom ${ }^{\circledR}$ Assay Design version 3.1 software (San Diego, CA, USA). The forward primer sequence was 5'-TTT CACCCATCTACAGTCCC-3', and reverse primer sequence was 5'-ACCTAGGCTCAGGGCAACTGACCG-3'. The PCR profile involved preliminary denaturation at an initial melting step of $95^{\circ} \mathrm{C}$ for $5 \mathrm{~min}$, followed by 35 cycles of denaturation at $95^{\circ} \mathrm{C}$ for $30 \mathrm{~s}, 55^{\circ} \mathrm{C}$ for $45 \mathrm{~s}$, and $72^{\circ} \mathrm{C}$ for $1 \mathrm{~min}$, with a final extension at $72^{\circ} \mathrm{C}$ for $10 \mathrm{~min}$. For quality control, approximately $10 \%$ of the samples were randomly selected from the cases and controls and were genotyped by PCR-RFLP. The results were $100 \%$ concordant.

\section{Statistical analysis}

All statistical analyses were performed using the SPSS version 11.0 software (SPSS 
Inc., Chicago, IL, USA) for Windows. Continuous variables are reported as means \pm standard deviation (SD) and were analyzed using the independent sample Student $t$-test. Categorical variables are reported as frequencies (percentages) and were analyzed using the $\chi^{2}$ test. The Kaplan-Meier method was used to estimate survival distribution, and the log-rank test was used to compare different survival times between patients with different genotypes. Cox proportional hazard regression was used to assess the association between polymorphisms in the $p 53$ gene and PFS and OS of NPC. All tests were two-sided, and $\mathrm{P}<0.05$ was considered to be statistically significant.

\section{RESULTS}

There were 127 patients included in the analysis, including 36 females and 91 males with a median age of $51.6 \pm 14.7$ years (31-76 years). Of the 127 patients, $116(91.1 \%)$ were WHO type III, and $40(31.8 \%)$ received chemotherapy (Table 1$)$.

\begin{tabular}{lcr}
\multicolumn{2}{c}{ Table 1. Demographic and clinical characteristics of NPC patients. } & $\%$ \\
\hline Characteristics & No. of patients (N =127) & \\
\hline Median age (years) & $51.6 \pm 14.7$ & 28.7 \\
Gender & 36 & 71.3 \\
$\quad$ Female & 91 & 8.9 \\
Male & & 91.1 \\
Histology & 11 & 68.2 \\
WHO type II & 116 & 31.8 \\
WHO type III & & \\
Chemotherapy & 87 & \\
No & 40 & \\
Yes & & \\
\hline
\end{tabular}

During the follow-up period, 42 patients died and 72 patients showed progression at the end of the study. Of the 127 patients, the median PFS time was $22.5 \pm 1.2$ months (1-36 months) and the median OS time was $28.2 \pm 1.1$ months (2-36 months).

The $p 53$ codon 72 Pro/Pro genotype was associated with a longer median PFS time of 30.3 months compared with 18.2 months for those with Arg/Arg variants [hazard ratio $(\mathrm{HR})=0.34,95 \%$ confidence interval $(95 \% \mathrm{CI})=0.10-0.97]$ (Table 2 and Figure 1$)$. Moreover, a significant association between $p 53$ codon 72 polymorphisms and PFS of NPC was observed in the recessive models, with an HR $(95 \% \mathrm{CI})$ of $0.37(0.12-0.99)$. However, we found no significant association between $p 53$ codon 72 polymorphisms and PFS of NPC in the dominant model.

Moreover, the $p 53$ codon 72 Pro/Pro genotype was associated with a longer median OS time of 31.6 months compared with 25.8 months for those with Arg/Arg variants, and the $\mathrm{P}$ value was marginally significant $(\mathrm{HR}=0.64,95 \% \mathrm{CI}=0.28-1.57)$ (Table 2 and Figure 2 ). However, no significant association was found between $p 53$ codon 72 polymorphisms and OS of NPC in the dominant and recessive models, with HRs (95\%CI) of $0.55(0.23-1.15)$ and $0.27(0.04-1.17)$, respectively. 
p53 codon 72 and nasopharyngeal carcinoma

10887

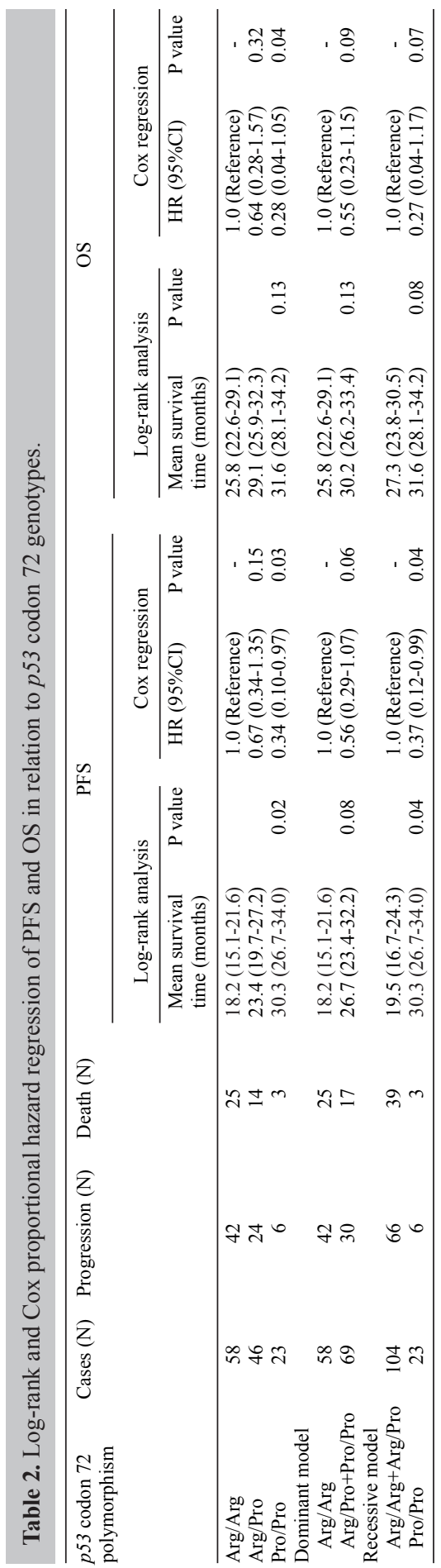




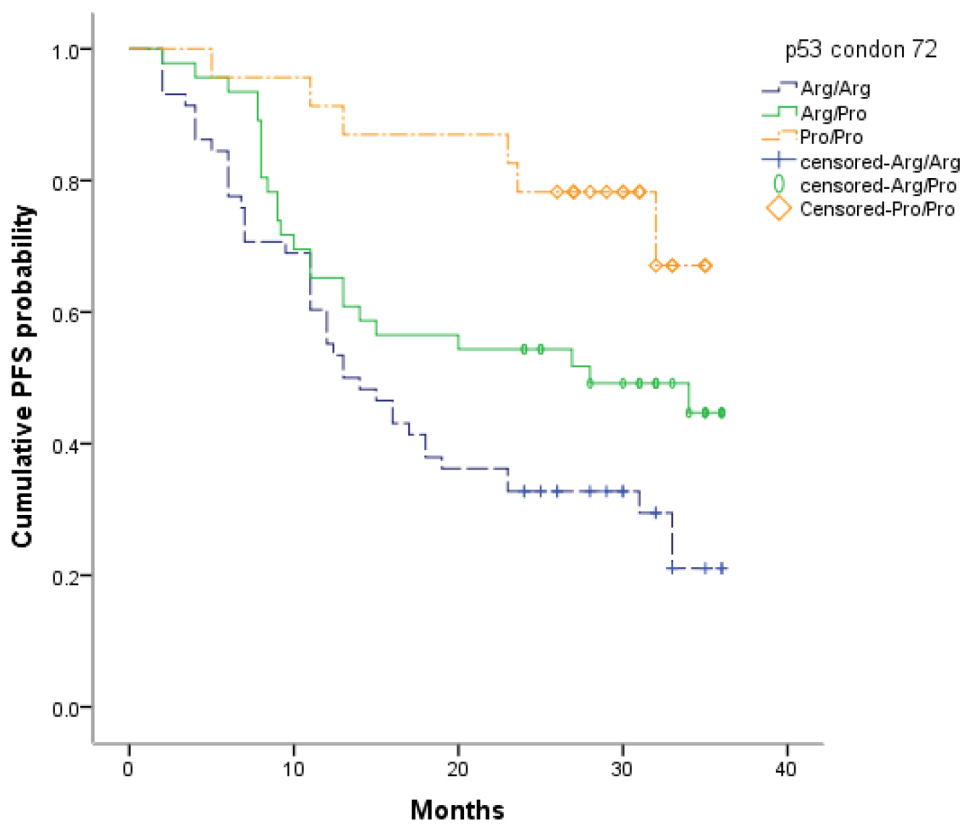

Figure 1. Kaplan-Meier analysis of PFS curves for NPC patients according to $p 53$ codon 72 genotypes.

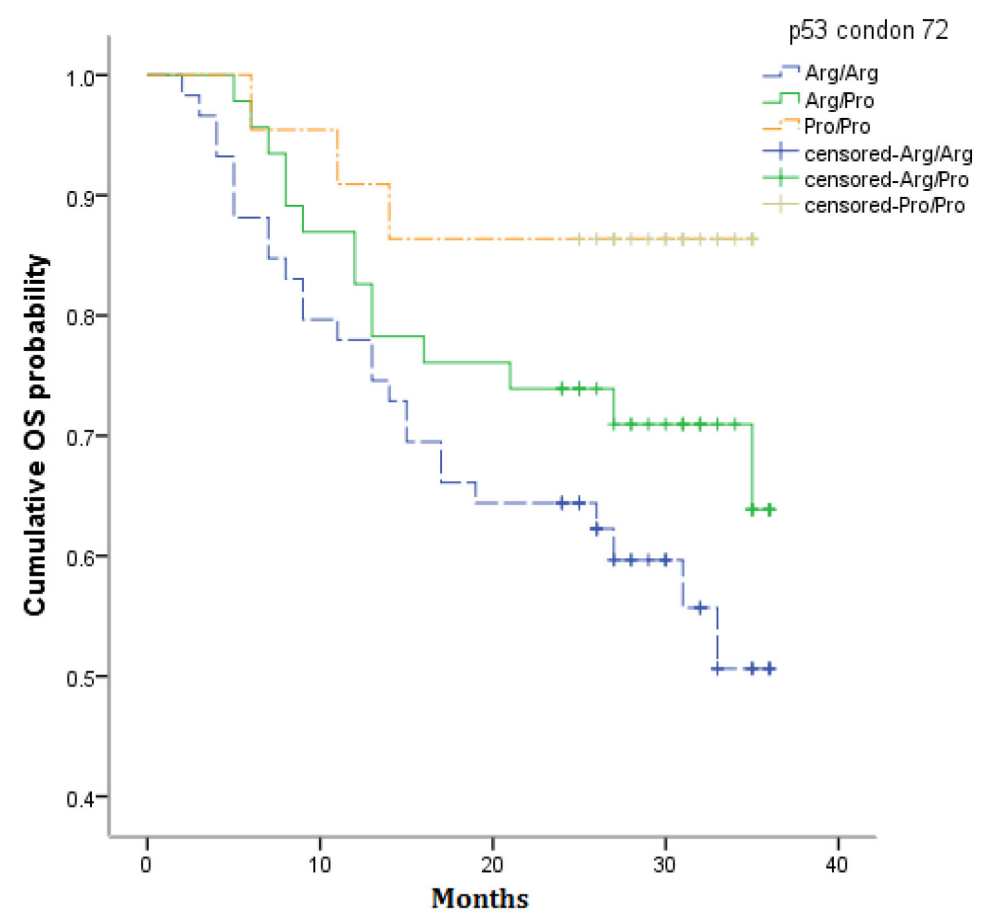

Figure 2. Kaplan-Meier analysis of OS curves for NPC patients according to $p 53$ codon 72 genotypes. 


\section{DISCUSSION}

In the current study, we found that patients carrying $p 53$ codon 72 Pro/Pro had longer PFS and OS compared with the Arg/Arg genotype. Cox proportional hazard regression and log-rank analysis indicated that genetic variations in $p 53$ codon 72 function as independent predictors of the clinical outcome of NPC receiving radiotherapy.

Variations in $p 53$ codon 72 reportedly alter the functional activity. Previous studies indicated that the $p 53$ codon 72 Pro/Pro genotype increased the ability to transactivate $\mathrm{p} 21$ and resulted in growth arrest (Thomas et al., 1999; Sullivan et al., 2004; den Reijer et al., 2008). Moreover, the $p 53$ codon $72 \mathrm{Arg} / \mathrm{Arg}$ variant showed superior mitochondrial localization in the tumor cell line, and this variant was more efficient in inducing apoptosis compared with p53 codon 72 Pro/Pro (Dumont et al., 2003). This high efficiency in inducing apoptosis results from the enhanced localization of the $p 53$ codon $72 \mathrm{Arg} / \mathrm{Arg}$ genotype to mitochondria (Dumont et al., 2003). Previous clinical studies indicated that $p 53$ codon 72 variants were associated with the clinical outcome of NPC (Alajez et al., 2009; Pan et al., 2009; Xie et al., 2013). Xie et al. (2013) reported that the $p 53$ codon 72 Pro/Pro genotype may be an effective independent prognostic marker for better outcome in patients with locoregional NPC, and positive protein expression and local regional lymph node metastasis can predict the progression of NPC. Pan et al. (2009) reported that variants in p53 improved radiotherapeutic tumor control survival rate in patients with NPC. However, the results are inconsistent (Ho et al., 2001; Agaoglu et al., 2004). Ho et al. (2001) showed that p53 overexpression did not significantly affect the locoregional response of primary tumor in patients with NPC receiving radiotherapy. Our study indicated that the $p 53$ codon 72 Pro/Pro genotype was associated with long PFS and OS among patients with locoregional NPC. The discrepancy in the results may be explained by the differences in the populations, study designs, and sample sizes. Further studies are needed to confirm the association between NPC polymorphisms and survival.

There were several limitations to our study. First, this study was conducted in a specific region of China, and the sample may not represent the population in other regions. Second, our study only examined $p 53$ codon 72 polymorphisms, and multiple genes may be involved in the clinical outcome of NPC; thus, other genetic factors and gene-gene correlations should be examined in future studies. Third, the number of NPC cases was relatively small, which may have reduced the statistical power in identifying differences between different genotypes. Therefore, further studies involving a larger sample size are needed to confirm the association between $p 53$ codon 72 polymorphisms and the clinical outcome of NPC.

In conclusion, we showed that variants in $p 53$ codon 72 may be independent predictors for PFS and OS of NPC. Our study suggests that genotyping of this polymorphism is helpful for predicting the clinical outcomes of patients with NPC. However, further studies with larger sample sizes are needed to confirm the predictive and prognostic role of p53 codon 72 polymorphisms in NPC.

\section{REFERENCES}

\footnotetext{
Agaoglu FY, Dizdar Y, Dogan O, Alatli C, et al. (2004). P53 overexpression in nasopharyngeal carcinoma. In Vivo 18: 555-560.

Alajez NM, Shi W, Hui AB, Yue S, et al. (2009). Targeted depletion of BMI1 sensitizes tumor cells to P53-mediated apoptosis in response to radiation therapy. Cell Death Differ. 16: 1469-1479.
} 
Baptiste N, Friedlander P, Chen X and Prives C (2002). The proline-rich domain of p53 is required for cooperation with anti-neoplastic agents to promote apoptosis of tumor cells. Oncogene 21: 9-21.

den Reijer PM, Maier AB, Westendorp RG and van Heemst D (2008). Influence of the TP53 codon 72 polymorphism on the cellular responses to X-irradiation in fibroblasts from nonagenarians. Mech. Ageing Dev. 129: 175-182.

Dumont P, Leu JI, Della Pietra AC, George DL, et al. (2003). The codon 72 polymorphic variants of p53 have markedly different apoptotic potential. Nat. Genet. 33: 357-365.

Fachiroh J, Sangrajrang S, Johansson M, Renard H, et al. (2012). Tobacco consumption and genetic susceptibility to nasopharyngeal carcinoma (NPC) in Thailand. Cancer Causes Control 23: 1995-2002.

Hildesheim A, Dosemeci M, Chan CC, Chen CJ, et al. (2001). Occupational exposure to wood, formaldehyde, and solvents and risk of nasopharyngeal carcinoma. Cancer Epidemiol. Biomarkers Prev. 10: 1145-1153.

Ho KY, Kuo WR, Chai CY, Tsai SM, et al. (2001). A prospective study of p53 expression and its correlation with clinical response of radiotherapy in nasopharyngeal carcinoma. Laryngoscope 111: 131-136.

International Agency for Research on Cancer (IARC) (2012). Globocan 2012: Estimated Cancer Incidence, Mortality and Prevalence Worldwide in 2012. IARC. Available at [http://globocan.iarc.fr/]. Accessed January 1, 2014.

Jia WH, Luo XY, Feng BJ, Ruan HL, et al. (2010). Traditional Cantonese diet and nasopharyngeal carcinoma risk: a largescale case-control study in Guangdong, China. BMC Cancer 10: 446.

Malkin D, Jolly KW, Barbier N, Look AT, et al. (1992). Germline mutations of the p53 tumor-suppressor gene in children and young adults with second malignant neoplasms. N. Engl. J. Med. 326: 1309-1315.

Pan JJ, Zhang SW, Chen CB, Xiao SW, et al. (2009). Effect of recombinant adenovirus-p53 combined with radiotherapy on long-term prognosis of advanced nasopharyngeal carcinoma. J. Clin. Oncol. 27: 799-804.

Polesel J, Franceschi S, Talamini R, Negri E, et al. (2011). Tobacco smoking, alcohol drinking, and the risk of different histological types of nasopharyngeal cancer in a low-risk population. Oral Oncol. 47: 541-545.

Song C and Yang S (2013). A meta-analysis on the EBV DNA and VCA-IgA in diagnosis of nasopharyngeal carcinoma. Pak. J. Med. Sci. 29: 885-890.

Sullivan A, Syed N, Gasco M, Bergamaschi D, et al. (2004). Polymorphism in wild-type p53 modulates response to chemotherapy in vitro and in vivo. Oncogene 23: 3328-3337.

Sun X, Zeng L, Chen C, Huang Y, et al. (2013). Comparing treatment outcomes of different chemotherapy sequences during intensity modulated radiotherapy for advanced $\mathrm{N}$-stage nasopharyngeal carcinoma patients. Radiat Oncol. 8: 265 .

Thomas M, Kalita A, Labrecque S, Pim D, et al. (1999). Two polymorphic variants of wild-type p53 differ biochemically and biologically. Mol. Cell Biol. 19: 1092-1100.

Xi M, Liu SL, Zhao L, Shen JX, et al. (2013). Prognostic factors and survival in patients with radiation-related second malignant neoplasms following radiotherapy for nasopharyngeal carcinoma. PLoS One 8: e84586.

Xie X, Wang H, Jin H, Ouyang S, et al. (2013). Expression of pAkt affects p53 codon 72 polymorphism-based prediction of response to radiotherapy in nasopharyngeal carcinoma. Radiat. Oncol. 8: 117. 